\title{
Exact states and spectra of vibrationally dressed polaritons
}

\author{
M. Ahsan Zeb, ${ }^{a}$ Peter G. Kirton, and Jonathan Keeling* \\ SUPA, School of Physics and Astronomy, University of St Andrews, St Andrews, KY16 \\ 9SS, United Kingdom \\ E-mail: jmjk@st-andrews.ac.uk
}

${ }^{a}$ Permanent address: National Centre for Physics, Islamabad 45320, Pakistan

\section{Abstract}

Strong coupling between light and matter is possible with a variety of organic materials. In contrast to the simpler inorganic case, organic materials often have a complicated spectrum, with vibrationally dressed electronic transitions. Strong coupling to light competes with this vibrational dressing, and if strong enough, can suppress the entanglement between electronic and vibrational degrees of freedom. By exploiting symmetries, we can perform exact numerical diagonalization to find the polaritonic states for intermediate numbers of molecules, and use these to define and validate accurate expressions for the lower polariton states and strong-coupling spectrum in the thermodynamic limit. Using this approach, we find that vibrational decoupling occurs as a sharp transition above a critical matter-light coupling strength. We also demonstrate how the polariton spectrum evolves with the number of molecules, recovering classical linear optics results only at large $N$.

\section{Keywords}

Organic microcavities, Strong coupling, lightmatter interaction, vibrational sidebands, Stokes shift.

Strong coupling between matter and light allows one to engineer material properties to in- duce desired collective behavior. A widely studied example of this is exciton polaritons in semiconductor microcavities. Strong coupling between microcavity photons and excitons produces quasiparticles with ideal properties for Bose condensation at elevated temperatures, ${ }^{1,2}$ allowing the study of interacting quantum fluids of light. ${ }^{3}$ Organic light emitting materials provide a particularly intriguing context in which to study the effects of strong coupling. Polaritons in such materials are stable up to room temperature, due to the high binding energy of Frenkel excitons and the large matter-light coupling strengths. ${ }^{4}$ Both strong coupling ${ }^{5-9}$ and polariton condensation or lasing ${ }^{10-14}$ have been seen in a wide variety of organic materials, including J-aggregates, small-molecule crystals, conjugated polymers, and even biologically produced materials.

Strong matter light coupling with organic materials also enables a range of further effects $^{15,16}$, in which material properties of the molecules such as chemical reaction rate, ${ }^{17-21}$ transport properties, ${ }^{22,23}$ superradiance, ${ }^{24}$ photochemistry, ${ }^{25}$ aspects of the molecular structure, ${ }^{26-28}$ or coupled electron-nuclear dynamics $^{29}$ can be modified by strong coupling of light to electronic transitions, or by coupling between infra-red cavities and optically active vibrational modes. ${ }^{30-34}$ Many of these effects have been associated with the way in which strong matter light coupling modifies the po- 
tential energy landscape for the conformation of the molecules. This also gives rise to the complex absorption and emission spectra observed in organic molecules, ${ }^{35}$ which often have large Stokes shifts, arising from a variety of photophysical effects. One significant contribution is the strong vibrational dressing of electronic transitions. The absorption spectrum is significantly modified in the presence of strong coupling to light, giving observable signatures of the change of vibrational conformation. In all these examples, the behavior observed results from collective strong coupling, with many molecules coupled to the same mode of light. Recently strong coupling has also been achieved in the limit of a small number of molecules, coupled to a plasmonic resonance ${ }^{36}$ highlighting the importance of developing theoretical techniques valid at finite molecule number.

In this paper, we consider the widely used Holstein-Tavis-Cummings model and give exact results for how strong matter-light coupling changes the vibrational configuration of the lower polariton state in this model. We show that there is a sharp crossover in the nature of the polaritonic ground state as one increases matter-light coupling. We find that vibrational decoupling only occurs above this critical strength, and so the resulting phenomena, such as enhanced electron transfer rate as discussed by Herrera and Spano ${ }^{18}$, only occur when the polariton splitting is sufficiently large. Most previous work addressing the competition of strong matter-light coupling and vibrational configuration have either relied on exact numerics for small numbers of molecules, or used physically motivated approximations without rigorous derivation. In contrast, we present exact numerical results for intermediate numbers of molecules, and derive analytic approximations which we show closely match the exact numerical results. This means we provide results that can cover the full range of molecule numbers from $N=1$ to the thermodynamic limit, $N \rightarrow \infty$. Indeed, using this approach we show that the polariton spectrum for small $N$ and large $N$ is quite distinct, and that linear optics results are only recovered in the large $N$ limit.

\section{Modeling organic polaritons}

Collective effects due to matter-light interaction have been studied extensively in the context of superradiance, lasing and polariton condensation. ${ }^{37}$ The archetypal models used to study this are the Tavis-Cummings ${ }^{38}$ and Dicke $^{39,40}$ models, which describe many twolevel systems coupled to light. However organic molecules are not two-level systems, and as discussed above, we must include the coupling between electronic states and molecular conformation, which produces vibrational sidebands in the absorption and emission spectra. In order to include such physics, we consider the Holstein-Tavis-Cummings (HTC) model which has been introduced and used in several previous works on strong coupling with organic molecules. ${ }^{18,27,28,41-43}$ Such a model allows one to find how strong matter-light coupling interacts with the formation of polarons - i.e. dressing of electronic states by vibrational excitations.

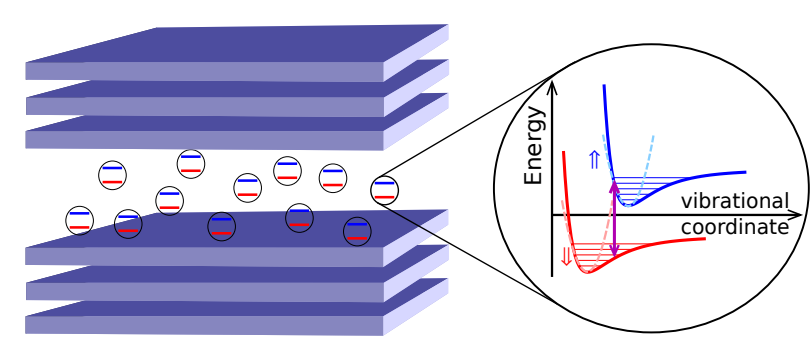

Figure 1: Cartoon of the Holstein-TavisCummings model, Eq. (1). We consider $N$ molecules placed in a cavity, where each molecule is represented by two electronic states, and an associated vibrational mode corresponding to the potential energy surface in the given electronic state.

The HTC model, illustrated in Fig. 1 is given by:

$$
\begin{array}{r}
\hat{H}=\omega \hat{a}^{\dagger} \hat{a}+\sum_{i=1}^{N}\left[\omega_{0} \hat{\sigma}_{i}^{+} \hat{\sigma}_{i}^{-}+\frac{\omega_{R}}{\sqrt{N}}\left(\hat{\sigma}_{i}^{+} \hat{a}+\hat{\sigma}_{i}^{-} \hat{a}^{\dagger}\right)\right. \\
\left.+\omega_{v}\left(\hat{b}_{i}^{\dagger} \hat{b}_{i}-\lambda_{0} \sigma_{i}^{+} \hat{\sigma}_{i}^{-}\left(\hat{b}_{i}^{\dagger}+\hat{b}_{i}\right)\right)\right] \cdot
\end{array}
$$

The cavity photon mode (annihilation operator $\hat{a}$ ) is coupled to many molecules, labeled 
$i=1 \ldots N$. Each molecule is described by two electronic states (Pauli operator $\hat{\sigma}_{i}$ ), and a (harmonic) vibrational degree of freedom (annihilation operator $\hat{b}_{i}$ ). The final term in the Hamiltonian reflects the offset between the optimal vibrational coordinate in the electronic ground state and in the excited electronic state. Specifically, the dimensionless parameter $\lambda_{0}$ measures the vibrational coordinate offset in units of the vibrational harmonic oscillator length (i.e. the size of the ground state vibrational wavefunction). The parameter $\lambda_{0}$ is related to the Huang-Rhys parameter $S$ via $S=\lambda_{0}^{2}$, which can be understood as the typical number of vibrational quanta excited in an electronic transition of the molecule (in the absence of strong coupling to light). The parameter $\lambda_{0}$ can also be related to the Stokes shift between the peak of the absorption spectrum and emission spectrum (in the absence of strong coupling to light). Roughly, the Stokes shift scales as $\omega_{v} \lambda_{0}^{2}$. The matter-light coupling (i.e. coupling between photons and electronic transitions of the molecules) is parameterized by the collective Rabi splitting $\omega_{R}$.

We work in the rotating wave approximation for this matter-light coupling, hence the number of electronic excitations plus photons, $N_{\text {ex }}=\hat{a}^{\dagger} \hat{a}+\sum_{i=1}^{N} \hat{\sigma}_{i}^{+} \hat{\sigma}_{i}^{-}$is conserved. We note that this approximation requires $\omega_{R}$ to be small with respect to the bare cavity and electronic transition frequencies, $\omega$ and $\omega_{0}$. Such an approximation is reasonable for many experiments, however there can exist experiments in the ultra-strong coupling regime where this approximation fails, and counter-rotating terms should be included. Since our focus in this paper is on the competition of matter-light coupling and the vibrational coupling, we focus on the regime where the rotating wave approximation is valid, as this allows direct comparison to previous results. ${ }^{18,43}$ While the RWA assumes the coupling $\omega_{R}$ is weak with respect to $\omega, \omega_{0}$, it does not place any restrictions on the size of the matter-light coupling as compared to the vibrational energy scales $\omega_{v}$ or $\omega_{v} \lambda_{0}^{2}$.

The vibrational dressing described by the HTC model is not unique to the organic molecular systems discussed here. Similar physics exists for color centers in diamond, ${ }^{44}$ and is also related to models studied in the context of optomechanics, where systems such as superconducting qubits act as a transducer between vibrational and optical modes. ${ }^{45,46}$ This connection between vibrationally dressed electronic transitions in molecules and optomechanics has been discussed by Roelli et al. ${ }^{47}$ The methods we present here can thus form the basis to answering other questions involving these classes of system (e.g. understanding the role of vibronic replicas of polaritons in condensation and lasing or finding polariton-polariton interactions), and our analytic ansatz gives new insights into the state of the $N$ molecule system.

\section{Results and discussion}

\section{Nature of lower polariton state}

In this section, we present the exact lower polariton state, and discuss its vibrational configuration. If one considers a single molecule, i.e. $N=1$ in Eq. (1), the physics of this model is well understood. Each sector of fixed $N_{\text {ex }}$ can be mapped to the Rabi model, describing a spin coupled to a bosonic mode. ${ }^{48-50}$ Our aim is to extend this understanding to large $N$. In what follows we take two approaches: (i) we describe how, even for relatively large values of $N$, it is possible to exactly diagonalize the Hamiltonian or time-evolve a state, and (ii) we compare these exact solutions to analytic approaches. The exact numerical results inform the analytic approaches in two ways: Firstly, studying the Wigner function of the exact solution, we can identify the nature of the eigenstates, and construct appropriate variational wavefunctions. Secondly, comparison of the exact and variational energies provides confirmation whether the analytic description is sufficiently accurate.

In order to identify the lower polariton state, we find the lowest energy eigenstate in the one excitation sector, $N_{\mathrm{ex}}=1$ and examine its vibrational configuration. We do this in Fig. 2 by showing the Wigner function (WF), $W(x, p)$ of the vibrational state of molecule $i$, conditioned 


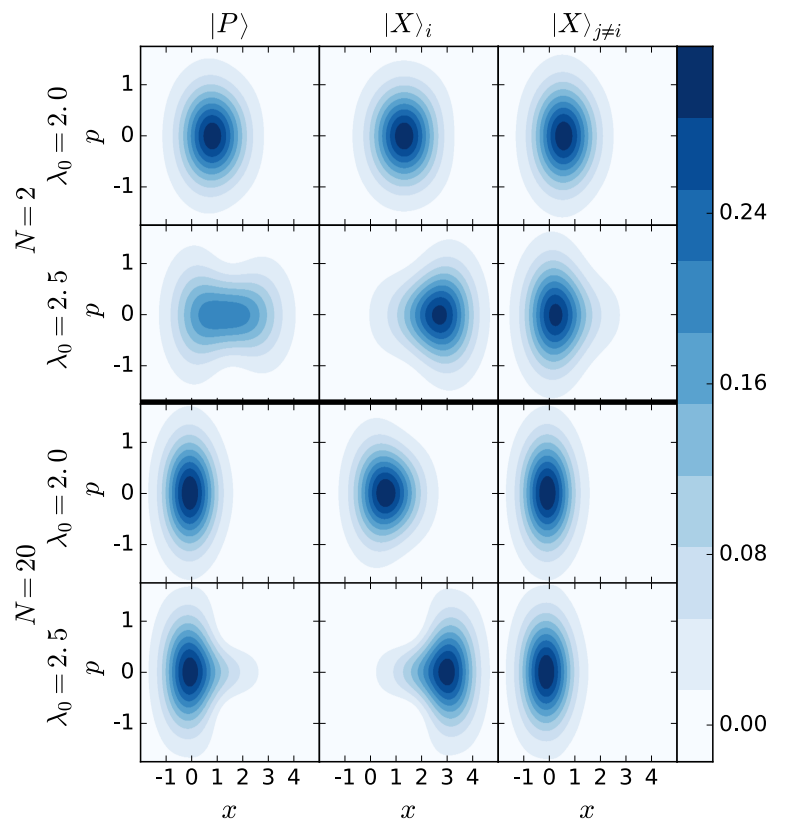

Figure 2: Conditional Wigner functions, $W_{|\psi\rangle}(x, p)$ for $N=2$ and $N=20$ at $\omega=\omega_{0}=$ $\omega_{R}=1, \omega_{v}=0.2$ and $\lambda_{0}$ as labeled. The first column is for a photon present in the cavity $|\psi\rangle=|P\rangle$, the second for the molecule $i$ being electronically excited $\left(|X\rangle_{i}\right)$, and the third for molecule $j \neq i$ being excited $\left(|X\rangle_{j \neq i}\right)$.

on one of three possible scenarios: a photon is present (labeled $|P\rangle$ ), the molecule $i$ is electronically excited $\left(|X\rangle_{i}\right)$, or another molecule, $j$ is excited $\left(|X\rangle_{j \neq i}\right)$. In plotting these figures, we normalise each Wigner distribution independently. The "total" Wigner function for a given molecular coordinate thus corresponds to a weighted sum of these conditional Wigner functions,

$$
\begin{gathered}
W(x, p)=\cos ^{2}(\theta) W_{|P\rangle}(x, p)+\sin ^{2}(\theta) \times \\
\frac{1}{N}\left[W_{|X\rangle_{i}}(x, p)+(N-1) W_{|X\rangle_{j \neq i}}(x, p) .\right] .
\end{gathered}
$$

Here the angle $\theta$ parameterizes the excitonphoton composition of the lower polariton state, and the factors of $N$ reflect the fact that the weight in the density matrix of a given molecule being excited is equal and there are $N-1$ molecules $j \neq i$. We show results for both $N=$ 2 and, a large number, $N=20$, and two values of $\lambda_{0}$, which, as discussed below are opposite sides of the decoupling crossover. Details of this exact numerical diagonalization are given in the methods section below. For $\lambda_{0}=2.0$ (rows 1 and 3 ), the vibrational WF is not strongly dependent on electronic state, and is close to the vacuum state (i.e. a Gaussian centered at $x=0, p=0)$. A small displacement is seen for the $|X\rangle_{i}$ state at $N=2$, but for $N=20$ this becomes very weak. For such a state, the vibrational and electronic states factorize, as discussed below. In the case $N=2$ the small displacement of the peak of the Wigner function away from $x=0$ can be associated to a small admixture of the excited state vibrational configuration. The suppression of this feature with increasing $N$ is discussed below in the context of the variational ansatz. For $\lambda_{0}=2.5$ (rows 2 and 4), quite distinct behavior is seen, despite the small change of $\lambda_{0}$. The vibrational and electronic state are strongly entangled: for $|X\rangle_{i}$ the vibrational state is very close to a displaced Gaussian. These exact results show that the vibrational decoupling discussed recently by Herrera and Spano ${ }^{18}$ occurs only for small $\lambda_{0}$. In that work it was shown that vibrational decoupling could have dramatic effects on electron transfer rates. From results below, we will see that the crossover value of $\lambda_{0}$ depends on matter light coupling, so that the enhancements of energy transfer can be expected to "turn on" above a critical value of matter-light coupling.

In addition to the vibrational decoupling, Fig. 2 for $N=2, \lambda_{0}=2.5$ shows another notable feature. We see $|P\rangle$ and $|X\rangle_{j \neq i}$ differ qualitatively - i.e. even when molecule $i$ is in its electronic ground state, its vibrational configuration depends on whether other molecules are excited. For $|P\rangle$ the vibrational WF is bimodal, while for $|X\rangle_{j \neq i}$ the vibrational WF is close to the vacuum state. The bimodal peak can be understood by considering potential energy surfaces vs vibrational coordinates, as discussed by Galego et al. ${ }^{27}$ : for $N=2$, within the lower polariton manifold the two dimensional vibrational potential energy surface as a function of $x_{i}=\left(\hat{b}_{i}^{\dagger}+\hat{b}_{i}\right) / \sqrt{2}$, has local minima at $\left(x_{1}, x_{2}\right)=\left(\sqrt{2} \lambda_{0}, 0\right),\left(0, \sqrt{2} \lambda_{0}\right)$. (Note that for $\omega=\omega_{0}$, there is no minima at $(0,0)$, so all local minima involve displacements of some co- 
ordinate.) The WF corresponds to a marginal distribution of the lower polariton wavefunction after integrating out the other coordinates. By conditioning on the electron and photon state, the WF becomes a biased version of this distribution. For $|X\rangle_{j \neq i}$, there is a bias toward $x_{j}=\sqrt{2} \lambda_{0}, x_{k \neq j}=0$, so the vibrational state for molecule $i \neq j$ is biased to 0 . For $|P\rangle$, this bias is absent, and the weights of the two minima are equal, giving a double peaked structure. On decreasing $\lambda_{0}$, or increasing $\omega_{R}$, the distinct minima merge, ${ }^{20}$ and the bimodal feature of the vibrational state vanishes replaced by a slightly displaced vacuum. The bimodality is not so visible for $N=20$. This is because the $N$ molecule system has $N$ potential minima, and the contribution of each minima is suppressed by $1 / N$. We show below that all of these features can be quantitatively recovered by an appropriate ansatz.

\section{Analytic approximation to lower polariton state}

The exact results shown in the previous system reveal how the vibrational decoupling varies significantly with parameter values, and how for the entangled state, the wavefunction takes a complicated form. These results are however costly to evaluate, and cannot be extended much beyond $N=20$. In this section, we use insight from these exact results to construct a variational wavefunction to recover the lower polariton state, and extrapolate our results from $N \lesssim 20$ to the thermodynamic limit, $N \rightarrow \infty$. We examine in turn a series of ansätze which approximately describe the lower polariton state including the vibrational mode. The most crucial feature displayed by Fig. 2 is the entanglement between the electronic and vibrational states when the coupling to vibrational modes is strong enough. To capture this we write the entangled polaron ansatz:

$$
\begin{gathered}
|\mathrm{EP}-\mathrm{F}\rangle=\alpha|P\rangle \otimes \prod_{j} \mathcal{D}\left(\lambda_{a}, \hat{b}_{j}\right)|0\rangle_{V} \\
+\frac{\beta}{\sqrt{N}} \sum_{i}|X\rangle_{i} \otimes \mathcal{D}\left(\lambda_{b}, \hat{b}_{i}\right) \prod_{j \neq i} \mathcal{D}\left(\lambda_{c}, \hat{b}_{j}\right)|0\rangle_{V}
\end{gathered}
$$

where $\mathcal{D}(\lambda, \hat{b})=\exp \left[-\lambda\left(\hat{b}^{\dagger}-\hat{b}\right)\right]$ is the vibrational displacement operator, and $|0\rangle_{V}$ is the vibrational vacuum state. This state has distinct vibrational configurations for each of $|P\rangle,|X\rangle_{i}$ and $|X\rangle_{j \neq i}$ shown in Fig. 2, allowing the required entanglement between vibrational and electronic state. The corresponding displacements are given by variational parameters, $\lambda_{a}, \lambda_{b}, \lambda_{c}$.

As Eq. (3) is linear in $\alpha, \beta$, one can easily minimize over these to find:

$$
E_{\mathrm{EP}-\mathrm{F}}=\frac{\omega_{X}+\omega_{P}}{2}-\sqrt{\tilde{\omega}_{R}^{2}+\left(\frac{\omega_{X}-\omega_{P}}{2}\right)^{2}},
$$

with

$\tilde{\omega}_{R}^{2}=\omega_{R}^{2} \exp \left[-\left(\lambda_{a}-\lambda_{b}\right)^{2}-(N-1)\left(\lambda_{a}-\lambda_{c}\right)^{2}\right]$,

a renormalized Rabi frequency, and

$$
\begin{aligned}
& \omega_{X}=\omega_{0}+\omega_{v}\left(\lambda_{b}^{2}-2 \lambda_{0} \lambda_{b}+(N-1) \lambda_{c}^{2}\right), \\
& \omega_{P}=\omega+\omega_{v} N \lambda_{a}^{2}
\end{aligned}
$$

are renormalized exciton and photon frequencies. We will consider two special cases of this ansatz, which we compare to the exact results in Fig. 3.

The first special case is to set $\lambda_{a}=\lambda_{b}=\lambda_{c} \equiv$ $\lambda$. This ansatz, which we refer to as the product polaron, $|\mathrm{PP}\rangle$, matches that in Herrera and Spano ${ }^{18}$. In this restricted ansatz, one finds that $\lambda \sim 1 / \sqrt{N}$, and so there is a decoupling between electronic and vibrational states. As seen in Fig. 3, this ansatz only becomes accurate when the Rabi frequency is very large compared to $\omega_{v} \lambda_{0}^{2}$ and hence is limited in the range of its applicability. The $|\mathrm{PP}\rangle$ ansatz works in this strong coupling limit because one can first diagonalize the matter-light problem, then 

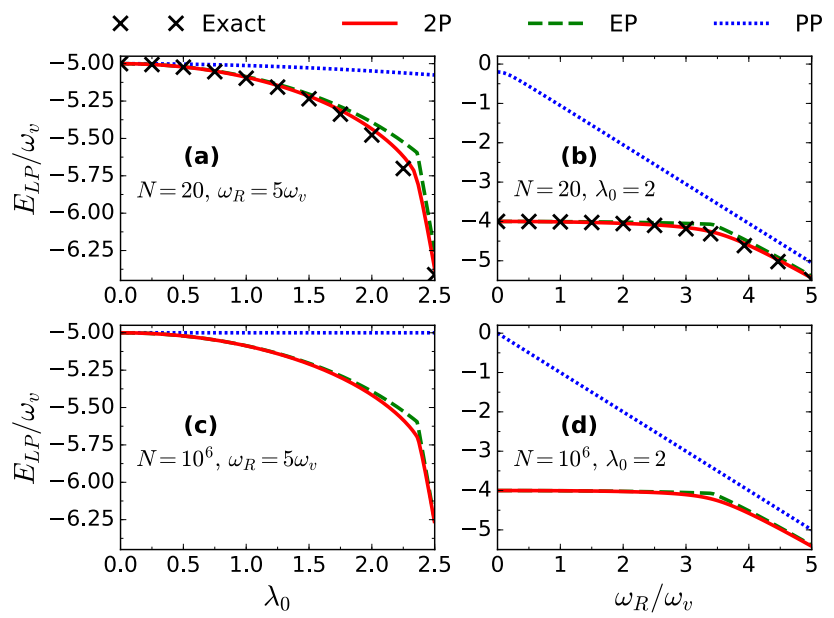

Figure 3: Energy vs $\lambda_{0}$ and $\omega_{R}$ for $N=20$ and $N=10^{6}$. The exact solution is shown as points, while various approximate expressions are shown as lines. $\omega_{0}=\omega$ for all panels, and is set as reference for $E_{L P}$.

treat the coupling to vibrations perturbatively. The matter-light problem splits into 'bright' polaritonic, and 'dark' states. The bright states are invariant under permutation of the electronic states of the molecules alone. In order to have entanglement between electronic and vibrational states, it is thus necessary to mix the bright and dark states, ${ }^{18}$ but when $\omega_{R} \gg \omega_{v} \lambda_{0}^{2}$, the large bright-dark splitting prevents vibrational coupling causing any mixing.

An improved restricted ansatz is based on considering the large $N$ limit, making no assumptions about other parameters. Minimizing the energy clearly requires non-zero $\tilde{\omega}_{R}$ and finite $\omega_{X}, \omega_{P}$. Hence, we may see that in the $N \rightarrow \infty$ limit one requires $\lambda_{a}=\lambda_{c}=0$, but we may keep non-zero $\lambda_{b} \equiv \lambda$. This state, $|\mathrm{EP}\rangle$, leads to an $N$-independent expression for $E_{\mathrm{EP}}$, which, as can be seen in Fig. 3, matches the exact energy well. The form of this ansatz makes clear why any displacement $\lambda_{a}$ of vibrational coordinates in the photon sector is suppressed at large $N$. For all parameters, the ansatz $|\mathrm{EP}\rangle$ is closer to the exact solution than $|\mathrm{PP}\rangle$. The only notable deviation between $|\mathrm{EP}\rangle$ and the exact solution occurs near $\omega_{R} \simeq \omega_{v} \lambda_{0}^{2}$, where there is a kink for $|\mathrm{EP}\rangle$ due to a crossing between the energies of the local minima near $\lambda \simeq 0$ and $\lambda \simeq \lambda_{0}$. The multiple minima for the $|\mathrm{EP}\rangle$ ansatz are illustrated in Figure 4.

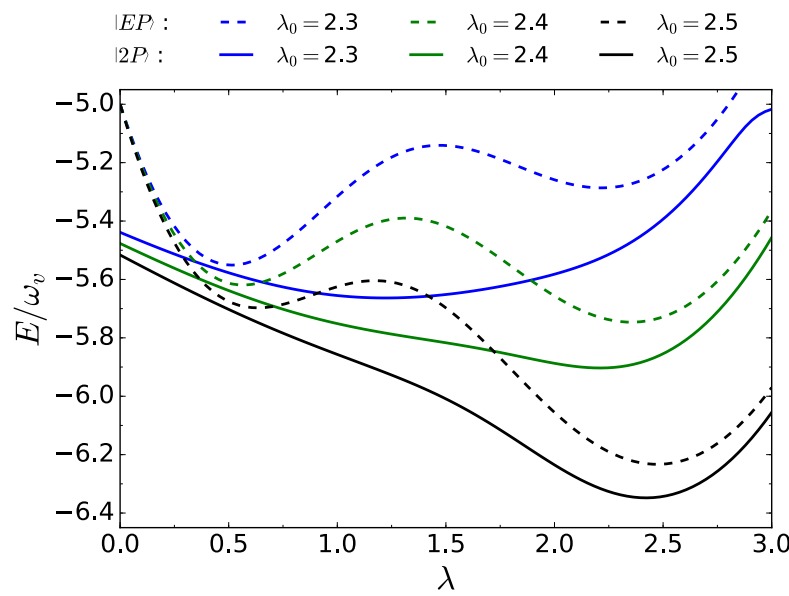

Figure 4: Energy landscape for $|\mathrm{EP}\rangle$ and $|2 \mathrm{P}\rangle$ as a function of variational parameter $\lambda$, plotted for $N=20$ and $\lambda_{0}$ as labeled. For $E P$, one sees there is a crossing between the local minima near $\lambda_{0}^{2} \simeq \omega_{R} / \omega_{v}$, while for $|2 \mathrm{P}\rangle$ this is smeared out.

As well as the sharp change in behavior visible from the ground state energy, a similar effect can be seen from the evolution of the photon fraction of the ground state, as shown in Fig. 5. In the limit of weak vibrational coupling, or strong Rabi splitting, we see the photon content of the lower polariton state approaches 0.5 , as expected for resonance between the photon and zero phonon line (i.e. $\omega=\omega_{0}$ ). When the vibrational coupling is strong or matter-light coupling is weak, the lower polariton instead becomes more excitonic, because the exciton energy including vibrational reorganization is $\omega_{0}-\omega_{v} \lambda_{0}^{2}$ which, for the parameters we show, is smaller than $\omega$.

To demonstrate that the change of characteristic behavior is indeed at $\omega_{R} \simeq \omega_{v} \lambda_{0}^{2}$, Fig. 6 plots the energy landscape of $|\mathrm{EP}\rangle$ vs the two relevant dimensionless parameters $\lambda_{0}$ and $\omega_{R} / \omega_{v}$ (the energy of $|\mathrm{EP}\rangle$ is independent of $N$ ). On this contour map, one can clearly see that the locus of the discontinuity follows this line. One may also note that a sharp kink exists for $\lambda_{0} \gtrsim 1.8$, otherwise the local minima in $E_{\mathrm{EP}}(\lambda)$ merge.

The failure of the $|\mathrm{EP}\rangle$ state close to the kink is similar to the failure of the polaron ansatz in the Rabi model. ${ }^{48,49}$ In the single molecule case, 


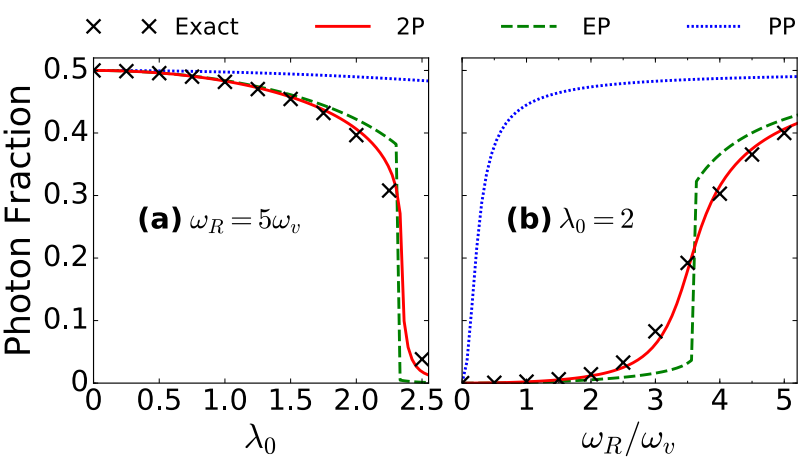

Figure 5: Photon content of the lower polariton vs $\lambda_{0}$ and $\omega_{R}$ for $N=20$ molecules. As in Fig. 3, the exact solution is shown as points, and the various approximations as lines. For the EP and PP ansätze, the photon fraction is given by $|\alpha|^{2}$ from Eq. (3). For the $2 \mathrm{P}$ ansatz, the photon fraction from Eq. (8) is $\alpha_{1}^{2}+2 \alpha_{1} \alpha_{2} e^{-\lambda^{2} / 2}+\alpha_{2}^{2}\left[1+(N-1) e^{-\lambda^{2}}\right] / N$. In terms of the overall Wigner function in Eq. (2), the photon fraction refers to $\cos ^{2}(\theta)$.

it is known this can be remedied by an ansatz including a superposition of two polaronic displacements. ${ }^{50}$ The exact Wigner function in this regime clearly shows a bimodal distribution, indicating such a multi-polaron ansatz is required. By considering the structure of the vibrational state revealed by the exact solution, we see that simultaneous displacement of multiple molecules is not significant. We therefore consider the following two polaron ansatz:

$$
\begin{gathered}
|2 \mathrm{P}\rangle=|P\rangle \otimes \frac{1}{N} \sum_{i}\left(\alpha_{1}+\alpha_{2} \mathcal{D}\left(\lambda, \hat{b}_{i}\right)\right)|0\rangle_{V} \\
+\frac{1}{\sqrt{N}} \sum_{i}|X\rangle_{i} \otimes\left(\beta_{1}+\beta_{2} \mathcal{D}\left(\lambda, \hat{b}_{i}\right)\right)|0\rangle_{V}
\end{gathered}
$$

This ansatz is more challenging to numerically minimize, information on the procedures we use are given in the methods section. As seen from the energy in Fig. 3, the $|2 \mathrm{P}\rangle$ ansatz has no kink at $\omega_{R} \simeq \omega_{v} \lambda_{0}^{2}$ and so matches the exact solution better than $|\mathrm{EP}\rangle$. This is explained in Fig. 4 which shows the energy landscape vs $\lambda$ for $|\mathrm{EP}\rangle$ and $|2 \mathrm{P}\rangle$ near this point. One sees that for $|\mathrm{EP}\rangle$ there are two minima, and so a change of characteristic behavior occurs when these minima cross. For $|2 \mathrm{P}\rangle$, the state is a

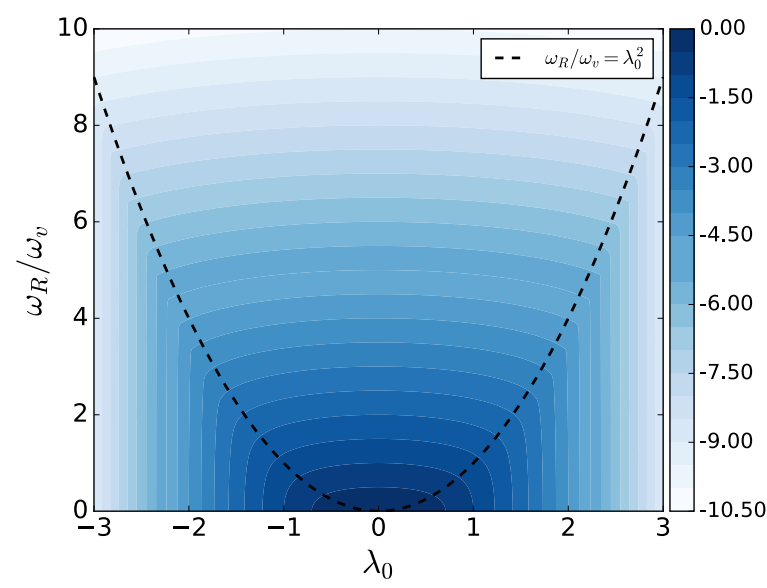

Figure 6: Optimized energy of $E_{\mathrm{EP}} / \omega_{v}$ as a function of $\lambda_{0}$ and $\omega_{R} / \omega_{v}$. The dashed line is the line $\omega_{R}=\omega_{v} \lambda_{0}^{2}$, and it can be seen that the locus of the discontinuity in energy closely follows this line at large $\lambda_{0}$, while at small $\lambda_{0}$, no discontinuity arises.

superposition of the two minima of $|\mathrm{EP}\rangle$, and so the distinct local minima are washed out.

Figure 7 shows the conditional Wigner functions for the same parameters as Fig. 2, but calculated from the $|2 \mathrm{P}\rangle$ ansatz. The $|2 \mathrm{P}\rangle$ ansatz assumes the $|X\rangle_{j \neq i}$ vibrational state to be the vacuum so the right column is always the same Gaussian. At $\lambda_{0}=2.0$ (rows 1 and 3 ), as seen in the exact results, the Wigner Function is close to the vibrational vacuum state almost independent of the electronic state. However, for $\lambda_{0}=2.5$ (rows 2 and 4), entanglement between the vibrational and electronic state is clearly visible. The ansatz reproduces the numerical results, including the suppression of entanglement with increasing $N$; this suppression occurs only when $\omega_{R}>\lambda_{0}^{2} \omega_{v}$, otherwise the variational parameter $\lambda$ saturates at $\lambda_{0}$ as $N$ increases. This demonstrates that $|2 \mathrm{P}\rangle$ captures not only the energy of the exact solution, but also captures well the exact state. i.e. Eq. (8) can be used to provide an accurate analytic description of the lower polariton state in future work.

\section{Absorption spectrum}

In the previous sections, we discussed the nature of the lower polariton state, with conse- 


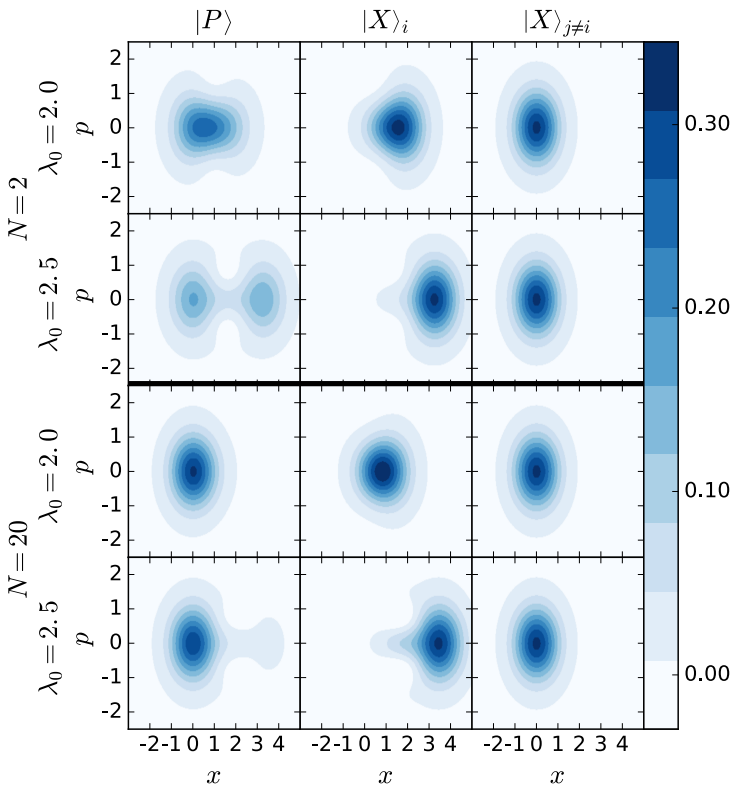

Figure 7: For $|2 \mathrm{P}\rangle$ ansatz, the conditional Wigner functions for $N=2$ (top) and $N=20$ (bottom) for the same parameters as in Figure 2.

quences on whether vibrational decoupling occurs. Here we show how the nature of this state is reflected by more routinely measured observables, specifically the absorption spectrum.

In the literature, there are two strands of work regarding how the strong coupling spectrum of vibrationally dressed excitons should be considered. In many works fitting experimental spectra, ${ }^{9,11-14}$ the vibrational sidebands of the molecule are treated as if they corresponded to separate species of emitters. i.e., one uses a "coupled oscillator" model, which describes a photon mode coupled to multiple excitonic resonances, one for each vibrational sideband. One can write such an approach both as a quantum Hopfield model, or as a classical linear optics calculation, with a material dielectric constant including all transitions from the ground state to vibrational sidebands, i.e. it assumes vibrational dressing of the electronic ground state is irrelevant. Given the results seen in Fig. 2 that indicate vibrational displacement for unexcited molecules, this result is concerning. An alternate approach is to consider diagonalising the full HTC model in the one excitation sector. Approximate methods for finding the spec- tra at intermediate $N$ have recently been discussed. ${ }^{51,52}$ Here we show how exact numerics at intermediate $N$ allows us to demonstrate the validity of simple approximate expressions valid in the thermodynamic limit, but show that more complicated results occur at small $N$.

Figure 8 shows how the absorption spec$\operatorname{trum}^{28,53} A(\nu)=-\kappa\left[\operatorname{Im}\left[D^{R}(\nu)\right]+\kappa\left|D^{R}(\nu)\right|^{2} / 2\right]$ evolves with number of molecules $N$ for three different values of $\lambda_{0}$. Here, $\kappa$ is the linewidth of cavity (with both mirrors assumed identical) and $D^{R}(\nu)$ is the photon Green's function. We show numerical results for intermediate $N$, and compare them to a calculation based on the coupled oscillator model. The analytic expression is given by writing $\left[D^{R}(\nu)\right]^{-1}=\nu+i \kappa / 2-\omega-\Sigma_{X}(\nu)$, where $\Sigma_{X}(\nu)$ is an excitonic self energy. In the large $N$ limit it is expected $28,41,54$ that this self energy can be calculated considering each molecule separately, leading to a simple form,

$$
\Sigma_{X}(\nu)=\sum_{m} \frac{\omega_{R}^{2}\left|f_{m}\right|^{2}}{\nu+i \gamma / 2-\left(\omega_{0}-\lambda_{0}^{2} \omega_{v}+m \omega_{v}\right)},
$$

where $f_{m}=\left\langle m\left|\mathcal{D}\left(\lambda_{0}, \hat{b}^{\dagger}\right)\right| 0\right\rangle$ is the overlap between the $m$ th excited vibrational state and the displaced ground state. This expression is clearly independent of $N$ (as the $1 / \sqrt{N}$ scaling of the matter-light coupling in Eq. (1) cancels with the sum over molecules).

As can be seen from Fig. 8, remarkably the analytic expression matches the exact results at $N \rightarrow \infty$, validating that such an expression applies in this limit, but clearly differs at small $N$.

To ensure convergence of the numerical results we include a much larger number of vibrational states, $M_{x}$, for the electronically excited molecule compared to the cutoff for electronically unexcited molecules at $M$. Both of these cutoffs vary with $N$. In particular, for small $N$, we find that more vibrational states must be kept. In all cases shown, convergence with increasing $M$ and $M_{x}$ was achieved, the smallest value used (for $N=30$ ) was $M=5$ and $M_{x}=20$.

The evolution of the spectral weight with in- 


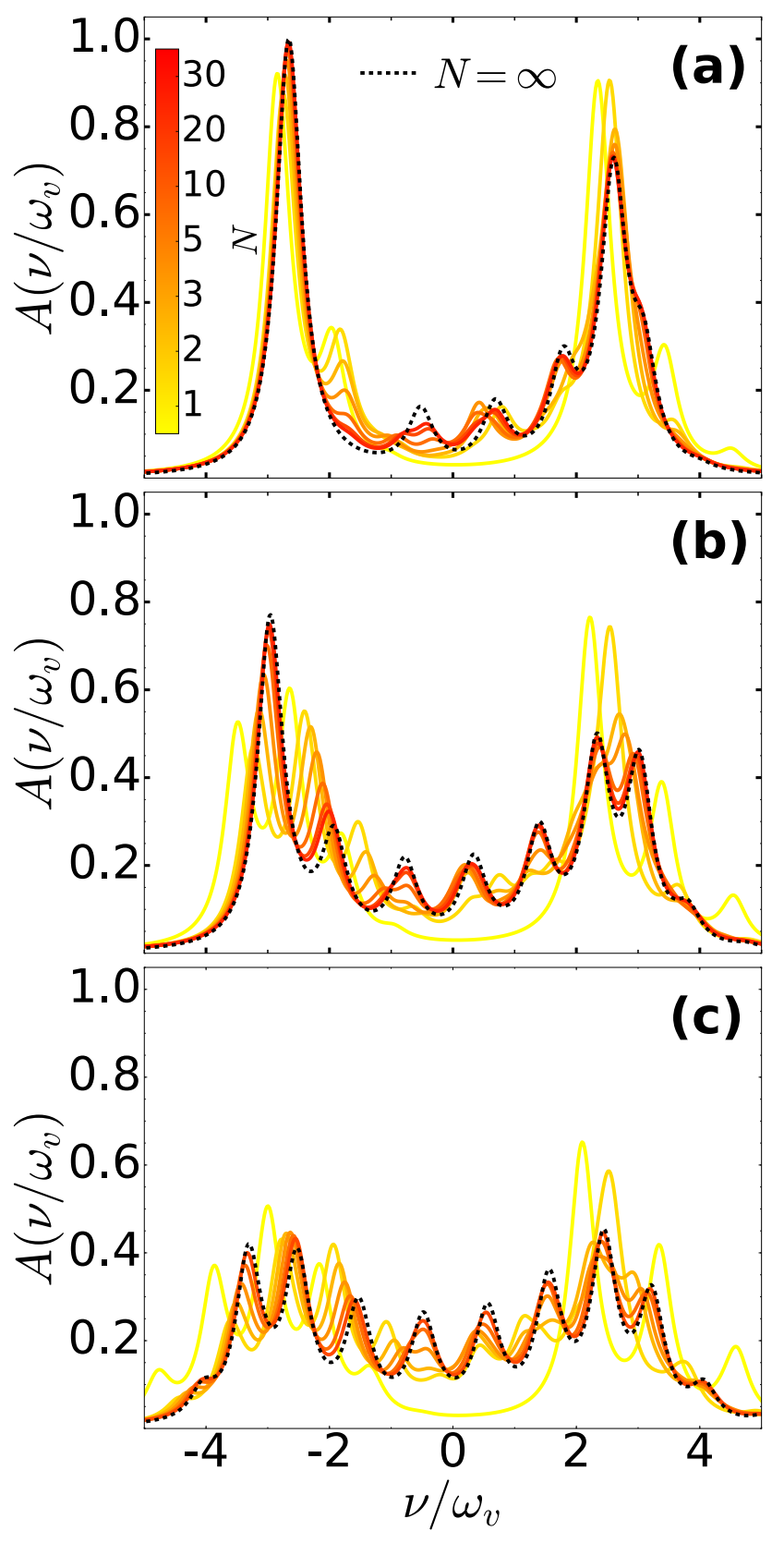

Figure 8: The evolution of absorption spectrum $A(\nu)$ with number of molecules $N$. We compare the exact solutions (solid) for varying number of molecules $N$, against the analytic Green's function (dotted) valid at $N \rightarrow \infty$ at $\omega_{R}=2.5 \omega_{v}$ and $\kappa=\gamma=0.5 \omega_{v}$ for three values of $\lambda_{0}=1,1.5,2$ in panels (a), (b), and (c) respectively. All curves are plotted for $M \geq 5$ and $M_{x} \geq 20$ as discussed in the text. creasing $N$ shows some interesting features. At small $\lambda_{0}$ when $N=1$ there is only a single vibrational replica close to the large lower and upper polariton peaks. As $N$ initially increases, other peaks grow, and then at very large $N$, several of the initially visible peaks vanish. The vanishing of peaks at large $N$, and hence the match to the coupled oscillator model, can be straightforwardly explained: these peaks involve transitions to vibrationally excited states in the electronic ground state manifold. Such states are absent from Eq. (9), as can be seen from the fact that this expression involves only transitions between the single state $|0\rangle$ in the ground state manifold, and a sequence of states in the excited manifold. For small $N$, transitions to such states have an effect, hence the small $N$ curves in Fig. 8 do not match Eq. (9). However, at large $N$, these transitions are suppressed. Physically, the Green's function corresponds to an amplitude to evolve from a state $|\alpha\rangle=a^{\dagger}|0\rangle \otimes|0\rangle_{V}$ back to $|\alpha\rangle$, i.e. to return back to a state without any vibrational excitations. Thus, any transition that excites a vibrational mode must be compensated by a subsequent transition (or transitions) returning a molecule to its vibrational ground state. However the probability that a single molecule is excited multiple times falls as a power of $N$. Hence, in the large $N$ limit, each molecule is excited only once, recovering Eq. (9). Similar behavior is seen as $\lambda_{0}$ increases. The competition between polaron and polariton physics makes all of the peaks similar in height.

\section{Methods}

\section{Exact numerical solution}

Here we focus on results that require only the $N_{\text {ex }}=1$ sector, but similar approaches allow extension to higher sectors. Within the $N_{\text {ex }}=1$ sector, possible states are spanned by the product between states

$$
\begin{aligned}
|X\rangle_{i} & =|0\rangle_{P}\left|\downarrow \ldots \uparrow_{i} \ldots \downarrow\right\rangle, \\
|P\rangle & =|1\rangle_{P}|\downarrow \ldots \downarrow\rangle,
\end{aligned}
$$


and vibrational states $\left|\left\{n_{i}\right\}\right\rangle_{V}$ (where $\left\{n_{i}\right\}$ count the vibrational quanta on each molecule).

Our numerical approach makes use of the fact that both the minimum energy state and the absorption spectrum of the system involve only states with full permutation symmetry between molecules. Such states, $|\Psi\rangle$ satisfy

$$
\begin{aligned}
& \left\langle\Psi \left|\left(|X\rangle_{j} \otimes\left|n_{1} \ldots n_{j} \ldots n_{k} \ldots n_{N}\right\rangle_{V}\right)\right.\right. \\
= & \left\langle\Psi \left|\left(|X\rangle_{k} \otimes\left|n_{1} \ldots n_{k} \ldots n_{j} \ldots n_{N}\right\rangle_{V}\right),\right.\right.
\end{aligned}
$$

for the excited molecule, along with symmetry under any permutation of the vibrational states of the $N-1$ unexcited molecules. Similarly the coefficients $\left\langle\Psi\left|\left(|P\rangle \otimes\left|\left\{n_{i}\right\}\right\rangle_{V}\right)\right.\right.$ have symmetry under permutations of all $n_{i}$. Among the symmetrically related coefficients, we can choose a single unique pattern $\left\{n_{i}\right\}$ and use this to represent all equivalent states. Truncating the number of vibrational quanta on a site to $n_{i} \leq M$, the symmetry reduces the problem size from a matrix of size $\mathcal{O}\left([M+1]^{N}\right)$ to $\mathcal{O}\left(N^{M}\right)$; i.e. from exponential in system size to polynomial. When $N$ is large, the basis size can still be kept to a manageable size without significantly compromising accuracy by using different number of vibrational states for electronically unexcited and excited molecules, $M$ and $M_{x}$ with $M_{x}>M$. Similar techniques have recently been used to study non-equilibrium steady states of related models. ${ }^{55}$ Full details of how to index these states is provided in the supplementary material ${ }^{1}$.

\section{Variational expressions}

Minimization of the variational ansätze are performed numerically. For the $|\mathrm{EP}\rangle$ ansatz the expression for the energy turns out to be simple. For the $|2 \mathrm{P}\rangle$, the result is more complicated. We first consider the normalization of the $|2 \mathrm{P}\rangle$ state. For the photonic part, one must be careful to include cross terms between different contributions in the sum, whereas for the excitonic part, entanglement with the electronic state means no cross terms exist. We will denote

\footnotetext{
${ }^{1}$ Supplementary material, containing details of the polynomial-scaling algorithm.
}

the cross term overlap as $\mathcal{O} \equiv\left\langle 0\left|e^{\lambda\left(\hat{b}_{i}^{\dagger}-\hat{b}_{i}\right)}\right| 0\right\rangle=$ $e^{-\lambda^{2} / 2}$, in terms of which we find:

$$
\begin{aligned}
& \langle 2 \mathrm{P} \mid 2 \mathrm{P}\rangle \\
=\frac{1}{N^{2}} \sum_{i j}\left[\alpha_{1}^{2}+2 \alpha_{1} \alpha_{2} \mathcal{O}\right. & \left.+\alpha_{1}^{2}\left(\delta_{i j}+\left(1-\delta_{i j}\right) \mathcal{O}^{2}\right)\right] \\
& +\beta_{1}^{2}+2 \beta_{1} \beta_{2} \mathcal{O}+\beta_{2}^{2} .
\end{aligned}
$$

This normalization condition can be written as a quadratic form in terms of the linear coefficients $\phi^{T}=\left(\alpha_{1}, \alpha_{2}, \beta_{1}, \beta_{2}\right)$. This means we may write, $\phi^{T} \mathbf{N} \phi=1$, where the matrix $\mathbf{N}$ in block form is:

$$
\begin{aligned}
\mathbf{N} & =\left(\begin{array}{cc}
\mathbf{N}^{\alpha} & 0 \\
0 & \mathbf{N}^{\beta}
\end{array}\right), \quad \text { where } \\
\mathbf{N}^{\alpha} & =\left(\begin{array}{cc}
1 & \mathcal{O} \\
\mathcal{O} & {\left[1+(N-1) \mathcal{O}^{2}\right] / N}
\end{array}\right), \\
\mathbf{N}^{\beta} & =\left(\begin{array}{cc}
1 & \mathcal{O} \\
\mathcal{O} & 1
\end{array}\right) .
\end{aligned}
$$

To calculate the energy, we split it in two parts. The non-vibrational terms (i.e. those from the Tavis-Cummings Hamiltonian) are relatively simple, as the photonic and electronic energies just follow the structure above, and the matter-light coupling term has the same matrix structure as the photon part, thus:

$$
E_{\mathrm{TC}}=\phi^{T} \mathbf{M}_{\mathrm{TC}} \phi, \quad \mathbf{M}_{\mathrm{TC}}=\left(\begin{array}{cc}
\omega \mathbf{N}^{\alpha} & \omega_{R} \mathbf{N}^{\alpha} \\
\omega_{R} \mathbf{N}^{\alpha} & \omega_{0} \mathbf{N}^{\beta}
\end{array}\right) .
$$

We must then add to this the vibrational part of the energy. This has the form:

$$
\frac{E_{v}}{\omega_{v}}=\frac{\alpha_{2}^{2} \lambda^{2}}{N}+\beta_{2}^{2}\left(\lambda^{2}-2 \lambda \lambda_{0}\right)-2 \beta_{1} \beta_{2} \mathcal{O} \lambda \lambda_{0} .
$$

Combining both parts we write $E_{2 \mathrm{P}}=\phi^{T} \mathbf{M} \phi$ where:

$$
\mathbf{M}=\left(\begin{array}{cc}
\omega \mathbf{N}^{\alpha}+\omega_{v} \mathbf{M}_{v}^{\alpha} & \omega_{R} \mathbf{N}^{\alpha} \\
\omega_{R} \mathbf{N}^{\alpha} & \omega_{0} \mathbf{N}^{\beta}+\omega_{v} \mathbf{M}_{v}^{\beta}
\end{array}\right),
$$


with the extra block matrices:

$$
\begin{aligned}
& \mathbf{M}_{v}^{\beta}=\left(\begin{array}{cc}
0 & -\lambda \lambda_{0} \mathcal{O} \\
-\lambda \lambda_{0} \mathcal{O} & \lambda^{2}-2 \lambda \lambda_{0}
\end{array}\right), \\
& \mathbf{M}_{v}^{\alpha}=\left(\begin{array}{cc}
0 & 0 \\
0 & \lambda^{2} / N
\end{array}\right) .
\end{aligned}
$$

In terms of the above expressions, the energy corresponds to minimization of $\phi^{T} \mathbf{M} \phi$ subject to the constraint $\phi^{T} \mathbf{N} \phi=1$. Thus the ground state energy corresponds to solving the generalized eigenvalue problem $\mathbf{M} \phi=\mu(\lambda) \mathbf{N} \phi$, and then minimizing over $\lambda$. We perform this minimization using a conjugate gradient approach from the SciPy library. ${ }^{56}$

It is instructive to note that at $N \rightarrow \infty$, there remains a regular form of all matrices above. This has the immediate consequence that at $N \rightarrow \infty$, we get a finite asymptote for the coefficients $\alpha_{1,2}, \beta_{1,2}$.

\section{Calculating retarded Green's functions}

The reflection, absorption and emission spectra of the coupled cavity-molecule system can all be found in terms of the retarded photon Green's function, ${ }^{28,52,53}$

$$
D^{R}(t)=-i \Theta(t)\left\langle 0\left|\left[\hat{a}(t), \hat{a}^{\dagger}(0)\right]\right| 0\right\rangle .
$$

In particular, the absorption spectrum $A(\nu)$ is given by

$$
A(\nu)=-\kappa_{L}\left[2 \operatorname{Im}\left[D^{R}(\nu)\right]+\left(\kappa_{L}+\kappa_{R}\right)\left|D^{R}(\nu)\right|^{2}\right],
$$

where $\kappa_{L, R}$ denote the losses through "left" and "right" mirrors of the cavity. In our plots, we assume a balanced cavity with $\kappa_{L}=\kappa_{R}$. The retarded Green's function can be found exactly by time evolution within the permutation symmetric states: i.e. one needs to find $\left\langle\alpha\left|e^{-i \hat{H} t}\right| \alpha\right\rangle$ where $|\alpha\rangle$ is a state with a single photon and all molecules in their electronic and vibrational ground state.

In evaluating this, it is useful to reduce the basis size by exploiting a vibrationally displaced basis set that lowers the vibrational cutoff $M$. In such a case, the vibrational part of the vac- uum state becomes a multidimensional coherent state.

To calculate a spectrum with finite linewidths, we incorporate decay rates for both the cavity, $\kappa$ and electronic excitations, $\gamma$, via non-Hermitian terms in the Hamiltonian. i.e. we write $H_{\text {decay }}=-i(\kappa|P\rangle\langle P|+\gamma| X\rangle\langle X|) / 2$ in the Hamiltonian for approximating the cavity and exciton decay rates, $\kappa$ and $\gamma$. Here, $|P\rangle\langle P|$ and $|X\rangle\langle X|$ are respectively projectors onto the subspace with a photon or exciton present.

\section{Summary}

In summary, we have presented exact solutions of the Holstein-Tavis-Cummings model at intermediate $N$, and used this to identify highly accurate analytic approaches which capture the behavior seen and can be extrapolated to $N \rightarrow$ $\infty$.

Regarding the nature of the lower polariton state, we find the behavior divides into two distinct classes: suppression of vibrational entanglement, and the consequent physics discussed in Ref. 18 when $\omega_{R} \gg \omega_{v} \lambda_{0}^{2}$, and a sharp crossover to entangled behavior when these scales become comparable. By considering the absorption spectrum, we have also shown how these entangled polaritonic states are nonetheless consistent with the previously used $^{28,41}$ form of absorption spectrum recovered in the $N \rightarrow \infty$ limit.

The method of exact solution we used applies to a variety of generalizations of the HolsteinTavis-Cummings model in the absence of disorder. For example, in future work, such exact solutions can be used to address the role of interactions or to go beyond the rotating wave approximation. The analytic ansatz presented provides an explicit description of the behavior of the model in the thermodynamic limit, and can provide a complementary tool to the methods proposed in Ref. 20. In the future, it may also provide a basis to develop the polaron master equation ${ }^{57-60}$ for $N$-molecule systems.

Acknowledgement JK and MAZ acknowledges financial support from EPSRC pro- 
gram "Hybrid Polaritonics" (EP/M025330/1). PGK acknowledges support from EPSRC (EP/M010910/1). We are grateful to F. Herrera, B. W. Lovett, and M. C. Gather for helpful comments on a previous version of the manuscript.

\section{References}

(1) Kasprzak, J.; Richard, M.; Kundermann, S.; Baas, A.; Jeambrun, P.; Keeling, J. M. J.; Marchetti, F. M.; Szymaska, M. H.; André, R.; Staehli, J. L.; Savona, V.; Littlewood, P. B.; Deveaud, B.; Dang, L. S. Bose-Einstein condensation of exciton polaritons. Nature 2006, 443, 409-14.

(2) Balili, R.; Hartwell, V.; Snoke, D.; Pfeiffer, L.; West, K. Bose-Einstein condensation of microcavity polaritons in a trap. Science 2007, 316, 1007-10.

(3) Carusotto, I.; Ciuti, C. Quantum fluids of light. Rev. Mod. Phys. 2013, 85, 299-366.

(4) Agranovich, V. M. Excitations in Organic Solids; Oxford University Press: Oxford, 2009.

(5) Lidzey, D. G.; Bradley, D.; Skolnick, M.; Virgili, T.; Walker, S.; Whittaker, D. Strong exciton-photon coupling in an organic semiconductor microcavity. Nature 1998, 395, 53-55.

(6) Lidzey, D. G.; Bradley, D. D. C.; Virgili, T.; Armitage, A.; Skolnick, M. S.; Walker, S. Room temperature polariton emission from strongly coupled organic semiconductor microcavities. Phys. Rev. Lett. 1999, 82, 3316.

(7) Lidzey, D. G.; Bradley, D. D.; Armitage, A.; Walker, S.; Skolnick, M. S. Photon-mediated hybridization of Frenkel excitons in organic semiconductor microcavities. Science 2000, 288, 1620-1623.

(8) Tischler, J. R.; Bradley, M. S.; Bulović, V.; Song, J. H.; Nurmikko, A.
Strong coupling in a microcavity LED. Phys. Rev. Lett. 2005, 95, 036401.

(9) Kéna-Cohen, S.; Davanço, M.; Forrest, S. R. Strong exciton-photon coupling in an organic single crystal microcavity. Phys. Rev. Lett. 2008, 101, 116401.

(10) Kéna-Cohen, S.; Forrest, S. Roomtemperature polariton lasing in an organic single-crystal microcavity. Nat. Phot. 2010, 4, 371-375.

(11) Plumhof, J. D.; Stöferle, T.; Mai, L.; Scherf, U.; Mahrt, R. F. Roomtemperature Bose-Einstein condensation of cavity exciton-polaritons in a polymer. Nat. Mater. 2014, 13, 247.

(12) Daskalakis, K. S.; Maier, S. A.; Murray, R.; Kéna-Cohen, S. Nonlinear interactions in an organic polariton condensate. Nat. Mater 2014, 13, 271-278.

(13) Michetti, P.; Mazza, L.; Rocca, G. C. L. In Organic Nanophotonics, Nano-Optics and Nanophotonics; Zhao, Y. S., Ed.; Springer, Berlin, 2015.

(14) Dietrich, C. P.; Steude, A.; Tropf, L.; Schubert, M.; Kronenberg, N. M.; Ostermann, K.; Höfling, S.; Gather, M. C. An exciton-polariton laser based on biologically produced fluorescent protein. Science Advances 2016, 2, e1600666.

(15) Flick, J.; Ruggenthaler, M.; Appel, H.; Rubio, A. Atoms and molecules in cavities, from weak to strong coupling in quantum-electrodynamics (QED) chemistry. Proceedings of the National Academy of Sciences 2017, 114, 3026-3034.

(16) Kowalewski, M.; Mukamel, S. Manipulating molecules with quantum light. Proceedings of the National Academy of Sciences 2017, 114, 3278-3280.

(17) Hutchison, J. A.; Schwartz, T.; Genet, C.; Devaux, E.; Ebbesen, T. W. Modifying chemical landscapes by coupling to vacuum fields. Ang. Chem. Int. Ed. 2012, 51, 1592-1596. 
(18) Herrera, F.; Spano, F. C. CavityControlled Chemistry in Molecular Ensembles. Phys. Rev. Lett. 2016, 116, 238301.

(19) Kowalewski, M.; Bennett, K.; Mukamel, S. Cavity Femtochemistry: Manipulating Nonadiabatic Dynamics at Avoided Crossings. J. Phys. Chem. Lett. 2016, 7, 2050-2054.

(20) Galego, J.; Garcia-Vidal, F. J.; Feist, J. Suppressing photochemical reactions with quantized light fields. Nat. Commun. 2016, 7, 13841.

(21) Galego, J.; Garcia-Vidal, F. J.; Feist, J. Many-Molecule Reaction Triggered by a Single Photon in Polaritonic Chemistry. Phys. Rev. Lett. 2017, 119, 136001.

(22) Orgiu, E.; George, J.; Hutchison, J. A.; Devaux, E.; Dayen, J. F.; Doudin, B.; Stellacci, F.; Genet, C.; Samori, P.; Ebbesen, T. W. Conductivity in organic semiconductors hybridized with the vacuum field. Nat. Mater. 2015, 14, 1123.

(23) Feist, J.; Garcia-Vidal, F. J. Extraordinary exciton conductance induced by strong coupling. Phys. Rev. Lett. 2015, 114, 196402.

(24) Zhang, Z.; Mukamel, S. Fluorescence spectroscopy of vibronic polaritons of molecular aggregates in optical microcavities. Chemical Physics Letters 2017, 683, 653 - 657, Ahmed Zewail (1946-2016) Commemoration Issue of Chemical Physics Letters.

(25) Bennett, K.; Kowalewski, M.; Mukamel, S. Novel photochemistry of molecular polaritons in optical cavities. Faraday Discuss. 2016, 194, 259-282.

(26) Schwartz, T.; Hutchison, J. A.; Genet, C.; Ebbesen, T. W. Reversible switching of ultrastrong light-molecule coupling. Phys. Rev. Lett. 2011, 106, 196405.
(27) Galego, J.; Garcia-Vidal, F. J.; Feist, J. Cavity-Induced Modifications of Molecular Structure in the Strong-Coupling Regime. Phys. Rev. X 2015, 5, 041022.

(28) Ćwik, J. A.; Kirton, P.; De Liberato, S.; Keeling, J. Excitonic spectral features in strongly coupled organic polaritons. Phys. Rev. A 2016, 93, 033840.

(29) Flick, J.; Appel, H.; Ruggenthaler, M.; Rubio, A. Cavity Born-Oppenheimer Approximation for Correlated ElectronNuclear-Photon Systems. Journal of Chemical Theory and Computation 2017, 13, 1616-1625, PMID: 28277664.

(30) Shalabney, A.; George, J.; Hutchison, J.; Pupillo, G.; Genet, C.; Ebbesen, T. W. Coherent coupling of molecular resonators with a microcavity mode. Nat. Comm. 2015, 6, 5981.

(31) del Pino, J.; Feist, J.; Garcia-Vidal, F. J. Quantum theory of collective strong coupling of molecular vibrations with a microcavity mode. New J. Phys. 2015, 17, 053040 .

(32) Shalabney, A.; George, J.; Hiura, H.; Hutchison, J.; Genet, C.; Hellwig, P.; Ebbesen, T. Enhanced Raman Scattering from Vibro-Polariton Hybrid States. Ang. Chem. Int. Ed. 2015, 54, 7971-7975.

(33) del Pino, J.; Feist, J.; Garcia-Vidal, F. J. Signatures of Vibrational Strong Coupling in Raman Scattering. J. Phys. Chem. C 2015, 119, 29132-29137.

(34) Strashko, A.; Keeling, J. Raman scattering with strongly coupled vibronpolaritons. Phys. Rev. A 2016, 94, 023843.

(35) Barford, W. Electronic and optical properties of conjugated polymers; Oxford University Press: Oxford, 2013.

(36) Chikkaraddy, R.; de Nijs, B.; Benz, F.; Barrow, S. J.; Scherman, O. A.; Rosta, E.; 
Demetriadou, A.; Fox, P.; Hess, O.; Baumberg, J. J. Single-molecule strong coupling at room temperature in plasmonic nanocavities. Nature 2016, 535, 127.

(37) Chiocchetta, A.; Gambassi, A.; Carusotto, I. In Universal Themes of BoseEinstein Condensation; Proukakis, N., Snoke, D., Littlewood, P., Eds.; Cambridge University Press, 2017; Chapter 20.

(38) Tavis, M.; Cummings, F. W. Exact Solution for an N-Molecule-Radiation-Field Hamiltonian. Phys. Rev. 1968, 170, 379.

(39) Dicke, R. H. Coherence in Spontaneous Radiation Processes. Phys. Rev. 1954, 93, 99-110.

(40) Garraway, B. M. The Dicke model in quantum optics: Dicke model revisited. Phil. Trans. R. Soc. A 2011, 369, 113755.

(41) Ćwik, J. A.; Reja, S.; Littlewood, P. B.; Keeling, J. Polariton condensation with saturable molecules dressed by vibrational modes. Europhys. Lett. 2014, 105, 47009.

(42) Spano, F. C. Optical microcavities enhance the exciton coherence length and eliminate vibronic coupling in $\mathrm{J}$ aggregates. J. Chem. Phys. 2015, 142, 184707.

(43) Wu, N.; Feist, J.; Garcia-Vidal, F. J. When polarons meet polaritons: Excitonvibration interactions in organic molecules strongly coupled to confined light fields. Phys. Rev. B 2016, 94, 195409.

(44) Doherty, M. W.; Manson, N. B.; Delaney, P.; Jelezko, F.; Wrachtrup, J.; Hollenberg, L. C. The nitrogen-vacancy colour centre in diamond. Phys. Rep. 2013, 528, 1-45.

(45) Pirkkalainen, J.-M.; Cho, S.; Massel, F.; Tuorila, J.; Heikkilä, T.; Hakonen, P.; Sillanpää, M. Cavity optomechanics mediated by a quantum two-level system. $\mathrm{Na}$ ture communications 2015, 6 .
(46) Heikkilä, T. T.; Massel, F.; Tuorila, J.; Khan, R.; Sillanpää, M. A. Enhancing Optomechanical Coupling via the Josephson Effect. Phys. Rev. Lett. 2014, 112, 203603.

(47) Roelli, P.; Galland, C.; Piro, N.; Kippenberg, T. J. Molecular cavity optomechanics as a theory of plasmon-enhanced Raman scattering. Nat. Nanotechnol. 2016, 11, 164.

(48) Irish, E. K.; Gea-Banacloche, J.; Martin, I.; Schwab, K. C. Dynamics of a twolevel system strongly coupled to a highfrequency quantum oscillator. Phys. Rev. B 2005, 72, 195410.

(49) Irish, E. K. Generalized Rotating-Wave Approximation for Arbitrarily Large Coupling. Phys. Rev. Lett. 2007, 99, 173601.

(50) Bera, S.; Nazir, A.; Chin, A. W.; Baranger, H. U.; Florens, S. Generalized multipolaron expansion for the spin-boson model: Environmental entanglement and the biased two-state system. Phys. Rev. B 2014, 90, 075110.

(51) Herrera, F.; Spano, F. C. Dark Vibronic Polaritons and the Spectroscopy of Organic Microcavities. Phys. Rev. Lett. 2017, 118, 223601.

(52) Herrera, F.; Spano, F. C. Absorption and photoluminescence in organic cavity QED. Phys. Rev. A 2017, 95, 053867.

(53) Ciuti, C.; Carusotto, I. Input-output theory of cavities in the ultrastrong coupling regime: The case of time-independent cavity parameters. Phys. Rev. A 2006, 74, 033811.

(54) Eastham, P. R.; Littlewood, P. B. Bose condensation of cavity polaritons beyond the linear regime: The thermal equilibrium of a model microcavity. Phys. Rev. B 2001, 64, 235101. 
(55) Kirton, P.; Keeling, J. Suppressing and Restoring the Dicke Superradiance Transition by Dephasing and Decay. Phys. Rev. Lett. 2017, 118, 123602.

(56) Jones, E.; Oliphant, T.; Peterson, P.; et al, SciPy: Open source scientific tools for Python. 2001; http://www.scipy.org/.

(57) McCutcheon, D. P. S.; Nazir, A. Quantum dot Rabi rotations beyond the weak excitonphonon coupling regime. New J. Phys. 2010, 12, 113042.

(58) McCutcheon, D. P. S.; Dattani, N. S.; Gauger, E. M.; Lovett, B. W.; Nazir, A. A general approach to quantum dynamics using a variational master equation: Application to phonon-damped Rabi rotations in quantum dots. Phys. Rev. B 2011, 84, 081305.

(59) Roy, C.; Hughes, S. Polaron master equation theory of the quantum-dot Mollow triplet in a semiconductor cavity-QED system. Phys. Rev. B 2012, 85, 115309.

(60) Pollock, F. A.; McCutcheon, D. P.; Lovett, B. W.; Gauger, E. M.; Nazir, A. A multi-site variational master equation approach to dissipative energy transfer. New J. Phys. 2013, 15, 075018. 


\section{Graphical TOC Entry}

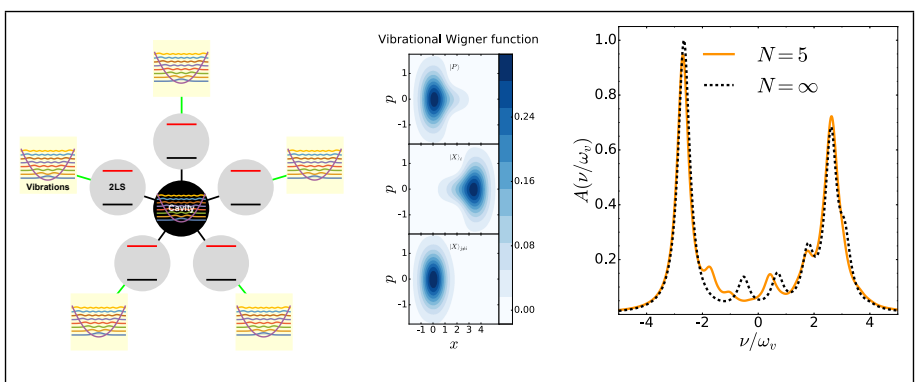

For Table of Contents Use Only

Exact states and spectra of vibrationally dressed polaritons M. Ahsan Zeb, Peter G. Kirton, and Jonathan Keeling

SUPA, School of Physics and Astronomy, University of St Andrews, St Andrews, KY16 9SS, United Kingdom

Brief description of TOC figure:

Left: graphical representation of the model used illustrated for five molecules: a single mode of the microcavity is coupled to the electronic transitions of all molecules, electronic states are in turn coupled to the intramolecular vibrational modes of respective molecules. Center: vibrational state of a molecule (shown by Wigner function $W(x, p)$ ) under three possible conditions, a photon is present $(|P\rangle$, top), an exciton is present at this molecule $\left(|X\rangle_{i}\right.$, middle), and an exciton is present at another molecule $\left(|X\rangle_{j \neq i}\right.$, bottom). Right: comparison of exact absorption spectrum $A\left(\nu / \omega_{v}\right)$ ( $\omega_{v}$ is the energy of a vibrational quanta) for a few molecules versus approximate analytical result in the thermodynamic limit. 